\title{
Factors Associated With Payments to Research Participants: A Review of Sociobehavioral Studies at a Large Southern California Research University
}

Journal of Empirical Research on

Human Research Ethics

2019, Vol. 14(4) 408-415

Do!: 10.1177/1556264619869538

journals.sagepub.com/home/jre

\author{
Brandon Brown', Logan Marg' (D, Zhiwei Zhang', \\ Dario Kuzmanović ${ }^{2}$, Karine Dubé ${ }^{3}$, and Jerome Galea ${ }^{4}$
}

\begin{abstract}
Along with a dearth of regulatory guidance, little empirical research has examined factors related to participant payment in research. We conducted a cross-sectional study of 100 institutional review board (IRB)-approved sociobehavioral human subjects research protocols at a large research university in Southern California. The proportion of studies that paid participants differed significantly by type of research $(p<.00 \mathrm{I})$ and study population $(p=.009)$. The average payment amount also differed significantly by study population $(p<.00 \mathrm{I})$ and type of participation (in-person vs. remote; $p<.00 \mathrm{I}$ ). In addition, studies that required more visits $(p<.00 \mathrm{I})$ and more time $(p=.0 \mathrm{II})$ paid significantly more than studies with fewer and shorter visits, respectively. These findings provide data to help inform future ethical payment practices.
\end{abstract}

\section{Keywords}

paying research participants, research ethics, institutional review boards

\section{Introduction}

Research participants often receive payment (e.g., cash, gift cards) and/or nonmonetary goods (e.g., course credit, small gifts) in return for their participation (Grady, 2005; Phillips, 2011). Researchers may choose to pay participants for various reasons (Gelinas et al., 2018), such as to bolster recruitment efforts (Bentley \& Thacker, 2004; Ripley, Macrina, Markowitz, \& Gennings, 2010b), compensate for studyrelated costs, time, and inconvenience (Fry et al., 2005; Mweemba, Ali, \& Hyder, 2018), and as a way to show appreciation for participants' contributions (Resnik, 2015). Research shows that amounts and forms of payment for research participation vary widely (Fry et al., 2005; Latterman \& Merz, 2001; Marathe et al., 2018). Some research also shows that payment amounts depend on the research setting, the amount of time that research participation requires, the procedures that participants will experience, and the type of research (e.g., early phase clinical experiments vs. late-phase clinical trials or biomedical vs. sociobehavioral research) (Fry et al., 2005; Marathe et al., 2018; Mweemba et al., 2018).

Despite the widespread practice of paying research participants, doing so remains ethically controversial; how much, when, and what to pay participants has been much debated (Beckford \& Broome, 2007; Phillips, 2011; Ripley,
2006; Wong \& Bernstein, 2011). Various models/approaches toward payment have also been described to explain and guide payment decisions (i.e., market, wage payment, reimbursement, appreciation) (Dickert \& Grady, 1999; Resnik, 2015; Ripley, 2006), yet determining appropriate payments for research participants remains a difficult endeavor.

The ethical issues of research participant payment mainly cluster around two major arguments. First, payments could result in undue inducement, whereby participants' assessment of the risks associated with research is altered by the payments (Resnik, 2015). In such cases, individuals' decisions to participate in research could be considered neither completely voluntary nor fully informed (Beckford \& Broome, 2007; Emanuel, Currie, Herman, \& Project, 2005; Macklin, 1981). Second, payments to research participants could result in biased recruitment by

\footnotetext{
'University of California, Riverside, USA

${ }^{2}$ University of Toronto, Ontario, Canada

${ }^{3}$ The University of North Carolina at Chapel Hill, USA

${ }^{4}$ University of South Florida, Tampa, USA
}

Corresponding Author:

Brandon Brown, Center for Healthy Communities, School of Medicine, University of California, Riverside, 3333 14th Street, Riverside, CA 9250I, USA.

Email: brandon.brown@medsch.ucr.edu 
encouraging higher enrollments of individuals with a lower socioeconomic status (SES) than individuals with a higher SES (Resnik, 2015). In these cases, payments to research participants could lead to an uneven distribution of the benefits and burdens of research, whereby research-related burdens mainly fall upon people of lower SES while people of higher SES are conferred the benefits (Denny \& Grady, 2007; Viens, 2001). Thus, payments to research participants could be exploitative, especially if participants are offered too little money in return for their contribution and efforts (Elliott \& Abadie, 2008). In addition, participants may conceal information to qualify for studies with payment, and in some cases even inflict self-harm to meet eligibility requirements (Devine et al, 2013; Lynch, Joffe, Thirumurthy, Xie \& Largent, 2019).

Despite the ubiquity of payments to research participants and their ethical complications, national and international research regulations and ethics guidelines require only that payments should neither be undue or exploitative, but provide little substantive guidance on how researchers and institutional review boards (IRBs) should determine appropriate payments (Council for International Organizations of Medical Sciences [CIOMS], 2016; Department of Health, Education, and Welfare \& National Commission for the Protection of Human Subjects of Biomedical and Behavioral Research, 2014). As a consequence, research institutions have needed to develop their own guidance for advising researchers on payment types and amounts, largely in the absence of empirical research to inform their policies. In particular, little is known about the practices of paying research participants, such as study factors that influence payment amounts and whether payments are offered (Brown et al., 2018). Data that could describe and characterize incentive types and amounts in actual studies could inform IRBs and similar regulatory bodies in their decision-making processes. The present study seeks to address this research gap by identifying the study factors associated with payments to research participants.

\section{Method}

We conducted a cross-sectional study of IRB-approved sociobehavioral research protocols and related materials (i.e., application forms, protocol amendments, informed consent forms, and correspondence between IRB officials and primary investigators) at a large research university in Southern California. The protocols were approved between January 2017 and January 2018. The university has a strong background in social sciences, humanities, engineering, and natural sciences. There were neither interactions nor discussions with investigators or participants of the reviewed studies. All study information unrelated to payment or other variables of interest were kept confidential. This study was exempt from IRB review as it did not involve interaction with study participants and did not collect identifiable data. An institutional official at the university provided permission and granted access to the de-identified administrative data.

The following data were collected from the study protocols and corresponding documents: (a) risk level (minimal or more than minimal) determined by the IRB; (b) IRB review type (full board, expedited, exempt); (c) expedited review category (1-7) (Office for Human Research Protections, 2016); (d) college/school of primary investigator; (e) number of participant visits; (f) participants' time commitment to the study (in minutes); (g) research type (qualitative, quantitative, or mixed methods); (h) whether the study participation was remote, in-person, or both; (i) population type (college students, nonstudent adults, children, or multiple); (j) whether payment was offered (something or nothing); (k) monetary payment amount (USD); and (l) payment form (money, academic credit, or other). The study's payment form was classified as "monetary" if any money was offered, "academic credit" if only academic credit was offered, and "other" if the payment involved a small gift, service, or nothing. Raffles were classified as "nothing," because virtually all study participants do not win raffles.

The number of visits was operationalized as any researcher-participant contact to include types of research that do not involve face-to-face contact, such as online surveys. In addition, because most study protocols specified a range of possible payments, visits, and time commitments for study participants, each of these variables was operationalized as the maximum possible.

\section{Statistical Analysis}

Chi-square and Student's $t$ tests were conducted to determine significant differences in the reported frequencies and means of variables between studies that offered payment and those that offered no payment. Univariate and multivariate linear regression models were used to examine the association between the amount of payment and other variables. The sandwich variance estimate was used to make inference in linear regression models without assuming normality or homoscedasticity. Analyses were conducted using Stata version 14.2 and R 3.5.2.

\section{Results}

Out of 117 available study protocols, 100 were included in our analysis. Of the 17 protocols excluded from the analysis, nine were unable to be located, which prevented data collection. The other eight protocols, most of which were secondary data analyses, were missing data on the variables of interest.

Out of the 100 included studies, all were sociobehavioral research: $73 \%$ of primary investigators were housed in the 
Table I. Factors Associated With Payments to Research Participants in 100 Social-Behavioral Studies.

\begin{tabular}{|c|c|c|c|c|}
\hline Group of studies & $\begin{array}{l}\text { Number } \\
\text { of studies }\end{array}$ & $\begin{array}{l}\text { Studies providing payment } \\
\qquad n(\%)\end{array}$ & $\begin{array}{l}\text { Payment amount (USD) } \\
\text { Mean| median (range) }\end{array}$ & $\begin{array}{l}\text { Hourly payment amount }{ }^{\mathrm{a}} \text { (USD/hr) } \\
\text { Mean| median (range) }\end{array}$ \\
\hline All & 100 & $55(55)$ & I5.92| $0.00(0-225)$ & $8.46 \mid 0.00(0-120)$ \\
\hline \multicolumn{5}{|l|}{ Visits $^{a}$} \\
\hline 1 & 66 & $36(54.5)$ & $8.20 \mid 0.00(0-100)$ & $9.70 \mid 0.00(0-120)$ \\
\hline 2 & 12 & $8(66.7)$ & $25.02 \mid \mathrm{I} .62(0-120)$ & $8.65 \mid 4.00(0-34)$ \\
\hline$>2$ & 20 & II (55.0) & $37.50 \mid 0.00(0-225)$ & $4.33 \mid 0.00(0-24)$ \\
\hline \multicolumn{5}{|l|}{ Time commitment $^{\mathrm{b}}$} \\
\hline $30 \mathrm{~min}$ or less & 22 & $16(72.7)$ & $4.67 \mid 0.75(0-30)$ & I5.99| $3.88(0-120)$ \\
\hline$>30 \mathrm{~min}$ to $60 \mathrm{~min}$ & 35 & $16(45.7)$ & $2.97 \mid 0.00(0-50)$ & $3.61 \mid 0.00(0-67)$ \\
\hline$>60 \mathrm{~min}$ & 40 & $23(57.5)$ & $34.62 \mid 0.00(0-225)$ & $8.56 \mid 0.00(0-50)$ \\
\hline \multicolumn{5}{|l|}{ IRB review type } \\
\hline Exempt & 5 & $2(40.0)$ & $3.40 \mid 0.00(0-15)$ & $10.50 \mid 6.00(0-30)$ \\
\hline Expedited & 93 & $52(55.9)$ & $16.66 \mid 0.00(0-225)$ & $8.43 \mid 0.00(0-120)$ \\
\hline Full review & 2 & I (50.0) & I2.50| I2.50 (0-25) & $5.77 \mid 5.77(0-12)$ \\
\hline \multicolumn{5}{|l|}{ Population type } \\
\hline Nonstudent adults & 58 & $25(43.1)$ & I5.29| $0.00(0-225)$ & $5.83 \mid 0.00(0-60)$ \\
\hline College students & 22 & $15(68.2)$ & $10.68 \mid 0.00(0-60)$ & $10.73 \mid 0.00(0-120)$ \\
\hline Multiple & 13 & $12(92.3)$ & $25.00 \mid 5.00(0-120)$ & $19.18 \mid 10.00(0-90)$ \\
\hline Children & 6 & $3(50.0)$ & $24.17 \mid 0.00(0-120)$ & $3.21 \mid 0.00(0-17)$ \\
\hline Organization & 1 & $0(.0)$ & $0.00 \mid 0.00(0-0)$ & $0.00 \mid 0.00(0-0)$ \\
\hline \multicolumn{5}{|c|}{ In-person and/or remote participation } \\
\hline In-person & 55 & $26(47.3)$ & $19.54 \mid 0.00(0-225)$ & $7.62 \mid 0.00(0-67)$ \\
\hline Remote & 27 & $19(70.4)$ & $3.95 \mid 0.25(0-50)$ & $10.82 \mid 1.00(0-120)$ \\
\hline Both & 18 & $10(55.6)$ & $22.79 \mid 2.50(0-120)$ & $7.53 \mid 1.29(0-50)$ \\
\hline \multicolumn{5}{|c|}{ Research method category } \\
\hline Quantitative & 46 & $32(69.6)$ & $|8.12| 0.62(0-225)$ & $11.64 \mid 3.16(0-120)$ \\
\hline Qualitative & 39 & II (28.2) & $7.31 \mid 0.00(0-120)$ & $2.94 \mid 0.00(0-34)$ \\
\hline Mixed & 15 & $12(80.0)$ & $33.43 \mid 6.00(0-200)$ & | 3.34| $4.88(0-67)$ \\
\hline
\end{tabular}

aExcluding two studies with missing visits.

${ }^{b}$ Excluding three studies with missing time.

College of Humanities, Arts, and Social Sciences (CHASS). Nearly all the studies (93\%) were deemed by the IRB to be expedited, which means they were no more than minimal risk and all procedures fell within the list of seven categories of expedited research published by Health and Human Services. Out of all 100 studies, only two were deemed to be more than minimal risk by the IRB. Most of the studies (91\%) fell under expedited review category 7 . In general, research that falls under expedited category 7 has no greater than minimal risk to participants and involves research. This category usually involves research on individual or group characteristics or behaviors or research employing surveys, interviews, oral histories, focus groups, program evaluation, human factors evaluation, or quality-assurance methodologies (Office for Human Research Protections, 2016).

Most studies (55\%) required in-person participation, $27 \%$ were conducted via the Internet, and $13 \%$ involved participants recruited via Amazon's Mechanical Turk marketplace (see Table 1). Study samples predominantly consisted of nonstudent adults (58\%) and college students $(22 \%)$. The number of study visits ranged from 0 to 34 (median $=1$ ), with an average of 2.8 visits. Time commitment for study participation ranged from $4 \mathrm{~min}$ to $300 \mathrm{hr}$, with an average of $5.6 \mathrm{hr}$ and a median of $1 \mathrm{hr}$. Most of the studies $(55 \%)$ offered some form of payment (e.g., cash, gift card, course credit, small gift, etc.). Among those, $41 \%$ of studies offered monetary payment, $53 \%$ offered something other than money (e.g., raffle or small gift) or nothing, and $6 \%$ offered academic credit. The average amount of monetary payment was USD 15.92 with a median amount of USD 0 . The amount of payment per hour ranged from USD 0 to 120, with an average of USD 8.46 per hour and a median of USD 0 per hour.

The proportion of studies that paid participants differed significantly $(p<.001)$ between qualitative $(11 / 39$ or $28 \%)$, quantitative (32/46 or $70 \%)$, and mixed-method (12/15 or $80 \%)$ studies. The proportion of studies that paid participants also differed significantly $(p=.009)$ between different study populations: nonstudent adults $(25 / 58$ or $43 \%)$, 
college students ( $15 / 22$ or $68 \%)$, and others $(15 / 20$ or $75 \%)$. Number of study visits, time commitment, review type, and type of participation (i.e., in-person, remote, etc.) were not significantly related to whether studies offered payment.

The following findings were generated from univariate linear regressions, where each variable was considered separately in relation to the amount of payment. The average payment amount differed significantly $(p<.001)$ between study populations: nonstudent adults (USD 15.29), college students (USD 10.68), children (USD 24.17), and others. Significant differences $(p=.008)$ were also found between types of participation: remote (USD 3.95), in-person (USD 19.54), and both (USD 22.79). Studies that required more visits paid significantly more than studies that required fewer visits $(p<.001)$, where each additional visit averaged an additional USD 4.29. Studies that required more time also paid significantly more than studies that required less time $(p=.011)$, with an estimated increase of USD 10.28 for a doubling of study time. There was a significant difference between the colleges/schools in which the studies' primary investigators were housed $(p<.0001)$; however, the small $p$-value is questionable given the small number of non-CHASS studies. Thus, we dichotomized studies into CHASS $(n=73)$ and non-CHASS $(n=27)$ and found no difference in amount of pay between those two groups. There also was not a significant relationship between amount of pay and study type (i.e., qualitative, quantitative, or mixed).

In a multivariate linear regression model, we used the dichotomized variable CHASS versus non-CHASS instead of each of the colleges/schools. In general, the covariates became less significant compared to univariate analyses. However, number of visits remained significant ( $p=.003)$, as did study population $(p=.033)$.

The amount of payment per hour was analyzed using similar (univariate and multivariate) linear regression models, and was found to be significantly $(p<.0001)$ associated with study population and research method category (quantitative, qualitative, or mixed) in univariate regression analyses. No significant associations were found in multivariate regression analyses of hourly payment.

\section{Discussion}

This study examined factors related to offers of payment and the amount of those payments in social-behavioral studies. There was a wide variation of payments across the studies, though the total amounts of monetary payments were modest (average USD 15.92). Quantitative studies were more likely to offer payment than qualitative studies, and mixed-method studies were more likely to offer payment than both qualitative and quantitative studies. The likelihood of offering payment also depended on study population, with nonstudent adults less likely to be paid than college students and other populations. Studies that required both in-person and remote participation paid more than studies that only required in-person or remote participation, and in-person participation paid more than remote participation. On average, studies involving children paid more than studies with college students and nonstudent adults, and nonstudent adults were paid more than college students. In addition, studies that required more visits and more time generally paid more than studies that required fewer visits and less time. However, when calculating the hourly payment rate (dollars per hour), studies with one visit and studies with the lowest time commitment generally paid a higher hourly rate. This may be due to limited budgets allotted to payment regardless of visits or time commitment.

The present study supports previous research which shows that payment levels in sociobehavioral research studies are relatively low, vary widely, and depend on the type of research, the amount of participation time required, the frequency of interactions between researchers and participants, and sample characteristics (Fry et al., 2005; Grady, Dickert, Jawetz, Gensler, \& Emanuel, 2005; Latterman \& Merz, 2001; Ripley, Macrina, Markowitz, \& Gennings, 2010a). Given that payment amounts tended to increase with more time required for participation and interactions between researchers and participants, our results are also consistent with previous research demonstrating that researchers tend to provide payments to participants to compensate them for their time and efforts (Fry et al., 2005; Mweemba et al., 2018).

The relatively low amounts of payment may be related to the university context in which the studies were conducted, where research is typically initiated by the primary investigator rather than other entities. For example, Marathe et al. (2018) found that studies initiated by investigators paid less than studies initiated by pharmaceutical companies or governments and suggested that budgetary constraints and investigator's perception of risks were likely the reasons for low payments in those studies. Therefore, budgetary constraints and investigators' perceptions of relatively low risk may have also contributed to low payment amounts. However, risks are usually not mentioned by regulatory guidelines as factors that should guide compensation amounts. The relatively low payments may also be due to the fact that all of the studies were sociobehavioral, which typically pay less than biomedical studies (Grady et al., 2005).

Our findings suggest mixed use of the practice of basing payment amounts on the hourly wage of an unskilled laborer, an approach recommended by numerous scholars (Dickert \& Grady, 1999; Grady, 2001; Shamoo \& Resnik, 2006). On one hand, the finding that more time and interactions with researchers were associated with higher payment is consistent with labor rules, which generally mandate that 
more work should equate to more pay (Ripley et al., 2010a). In addition, the finding that nonstudent adults were paid more than college students may indicate that researchers considered the value of respective participants' wage potential, where nonstudent adults generally earn more than college students and were therefore paid more (Dickert \& Grady, 1999). Conversely, the relatively high average time of participation $(5.6 \mathrm{hr}$ ) and low average payment (USD 15.92) across the studies is inconsistent with the wage-payment model, as is the fact that payment for studies with children was higher than that with adults of either type. Thus, our findings are indicative of the functionality of the wagepayment model "on the ground," where certain elements of the model (e.g., paying more for more work) appear more feasible than others (e.g., paying a standard hourly wage) (Ripley et al., 2010a). Moreover, the relatively low levels of payment and risk suggest that payments were not undue, which is consistent with previous research (Latterman \& Merz, 2001; Ripley et al., 2010a).

The finding that studies involving children paid more than studies involving nonstudent adults and college students is interesting, particularly in light of the ethical debate surrounding research payments to children (Bagley, Reynolds, \& Nelson, 2007; Iltis, Matsuo, \& DeVader, 2008; Ross, 2005; Wendler, Rackoff, Emanuel, \& Grady, 2002). Specifically, there are concerns that children are more susceptible to undue influence from payment, and have limited capacity to understand the requirements of participation and the potential risks of participation (Taplin et al., 2019). However, considering that nearly all of our data came from minimal risk studies and the relatively low payment levels (the highest being USD 120 for a 46.5-hr study across 14 weeks), it is unlikely that child research participants were unduly influenced (Taplin et al., 2019; Wendler et al., 2002). In addition, research suggests that children are not at greater risk for undue inducement than other populations and that payments can be utilized to increase children's participation in research without concerns of undue inducement as long as they are age-appropriate (Bagley et al., 2007; Bentley \& Thacker, 2004; Taplin et al., 2019). Higher payments in studies involving children in the present study may have been due to increased difficulty accessing and recruiting those populations, as well as the additional requirement of parent involvement for transportation, waiting time, and other potential assistance for their child to complete the study.

Our findings are supportive of the contention that concerns about undue influence have been overemphasized and that considering whether payments are too high should be secondary to considering whether payments are so low that they are exploitative (Emanuel, 2005; Largent \& Lynch, 2017). In fact, due to unfounded concerns about undue influence, payment can conceivably be higher in low-risk research such as in the cross-sectional study, depending on individual study budgets. That said, eliminating unnecessary risks, especially excessive risks of harm, and mitigating those that persist are fundamental responsibilities among researchers, ethics committees, and related entities independent of any payment. By exercising those responsibilities, concerns about undue influence are largely eliminated (Emanuel, 2005; Largent \& Lynch, 2017; Singer \& Couper, 2008).

\section{Best Practices}

The wide variation of payment practices across studies in the same region and populations suggests that decisionmaking for payment in research is haphazard. As such, we recommend that study authors describe their reasoning behind the payments they provided in their peer-reviewed manuscripts. Doing so would provide a starting point for gathering more data on how payment decisions are made. Currently, rationales behind payments are seldom, if ever, reported, and few institutions may have formal and standard payment policies based on study type and contextual factors of the research setting. Understanding how payment decisions are made is important for generating practical guidance.

This study's findings are limited in several ways. First, no statistical adjustments were made for multiple testing; therefore, the statistical significance of the reported associations may have been overly interpreted. However, all associations in research should be interpreted with attention to statistical adjustments. Second, the sample size was relatively small. The study protocols were all submitted in the same year and conducted at the same university. Thus, the findings may not generalize to other institutions or contexts. However, this was one of the first reviews of payment paired with study-related data which can give us a sense of how payment decisions are made. Third, all the studies were sociobehavioral, so the findings may not generalize to biomedical research, which is usually higher risk. However, as we were able to identify differences in lower risk studies, differences may also exist among higher risk studies. Finally, no studies differentiated between reimbursement for costs, compensation for time and burdens, and incentives for participation, which is inconsistent with a recent framework for providing ethical payments to research participants (Gelinas et al., 2018). Indeed, the words "incentive" and "compensation" were often used interchangeably in study protocols and the rationale behind payments was seldom articulated. As such, we were unable to identify the rationales behind decisions to pay research participants in these studies. However, the current study provides rationale for specifying the exact payment type in future research to overcome this challenge. 
Table 2. Key Findings and Considerations.

- Our findings suggest that payment decisions are made by study type, participation type, participant type, time, and number of visits. - More time and more visits resulted in more monetary payments.

- Budgetary constraints and investigators' perceptions of relatively low risk may have contributed to low payment amounts. 0 Risks are usually not mentioned by regulatory guidelines as factors that should guide payment amounts.

- Certain elements of the wage-payment model, such as paying more for more work, appear more feasible than others, such as paying a standard hourly wage.

- The relatively low levels of payment and risk suggest that payments were neither undue nor coercive.

- Our findings support the idea that concerns about undue influence have been overemphasized and that considering whether payments are too high should be secondary to considering whether payments are so low that they are exploitative.

- Fewer than half of the studies provided monetary payment, so underpayment may be a concern for participants' time.

- Quantitative studies tended to provide higher payment compared to qualitative or mixed-methods studies.

\section{Research Agenda}

Future research should compare payment amounts in minimal risk and above minimal risk studies to investigate the influence of study risk on payments to research participants. In addition, future research should compare payment for similar studies in different locations to examine the role of institutional and geographic context on payment practices. Any research agenda should have the goal to develop guidance for payment in research.

\section{Educational Implications}

Guidance for payment in research should be provided as part of IRB applications. Doing so would enable key stakeholders in the research process-including funders, policy makers, study participants, ethics committee members, and researchers - to critically assess how payment decisions are made, how they report payment data, and how data can be used to develop payment policy. Our data alone are a sample of mostly minimal risk sociobehavioral studies at a single institution, and not meant to create standards of payment. More data from multiple institutions with studies of varying risk are needed.

\section{Conclusion}

Despite its limitations, this study is one of few that have analyzed study factors related to payments to research participants. Even fewer studies have examined payments in sociobehavioral research. We hope the findings will inform scholarly debate about the ethics of payments to research participants as well as guidelines for payment practices (see Table 2 for a summary of key findings and considerations).

\section{Acknowledgments}

The authors would like to thank the institutional review board (IRB) analysts, Monica Wicker and Lorraine Castro, who assisted with and provided access to the data. They would also like to thank Jo Gerrard for editorial assistance.

\section{Declaration of Conflicting Interests}

The author(s) declared no potential conflicts of interest with respect to the research, authorship, and/or publication of this article.

\section{Funding}

The author(s) disclosed receipt of the following financial support for the research, authorship, and/or publication of this article: This study was supported in part by a National Institutes of Health diversity supplement (3R01AI114617-03S1).

\section{ORCID iD}

Logan Marg (iD https://orcid.org/0000-0003-0547-075X

\section{References}

Bagley, S. J., Reynolds, W. W., \& Nelson, R. M. (2007). Is a "wage-payment" model for research participation appropriate for children? Pediatrics, 119(1), 46-51. doi:10.1542/ peds.2006-1813

Beckford, L., \& Broome, M. R. (2007). Ethics and the payment of research subjects. Psychiatry, 6, 83-85. doi:10.1016/j. mppsy.2006.11.001

Bentley, J. P., \& Thacker, P. G. (2004). The influence of risk and monetary payment on the research participation decision making process. Journal of Medical Ethics, 30, 293-298. Retrieved from https://www.ncbi.nlm.nih.gov/pubmed/15173366

Brown, B., Galea, J. T., Dube, K., Davidson, P., Khoshnood, K., Holtzman, L., . . . Taylor, J. (2018). The need to track payment incentives to participate in HIV Research. IRB: Ethics \& Human Research, 40(4), 8-12. Retrieved from https://www .ncbi.nlm.nih.gov/pubmed/30387975

Code of Federal Regulations Title 21, Part 50 (2017).

Council for International Organizations of Medical Sciences. (2016). International ethical guidelines for health-related research involving humans (4th ed.). Geneva, Switzerland: Author.

Denny, C. C., \& Grady, C. (2007). Clinical research with economically disadvantaged populations. Journal of Medical Ethics, 33, 382-385. doi:10.1136/jme.2006.017681

Department of Health, Education, and Welfare \& National Commission for the Protection of Human Subjects of Biomedical and Behavioral Research. (2014). The Belmont 
Report: Ethical principles and guidelines for the protection of human subjects of research. Journal of the American College of Dentists, 81(3), 4-13. Retrieved from https://www.ncbi .nlm.nih.gov/pubmed/25951677

Devine, E. G., Waters, M. E., Putnam, M., Surprise, C., O’Malley, K., Richambault, C., . . . Ciraulo, D. A. (2013). Concealment and fabrication by experienced research subjects. Clinical Trials, 10, 935-948. doi:10.1177/1740774513492917

Dickert, N., \& Grady, C. (1999). What's the price of a research subject? Approaches to payment for research participation. The New England Journal of Medicine, 341, 198-203. doi:10.1056/NEJM199907153410312

Elliott, C., \& Abadie, R. (2008). Exploiting a research underclass in phase 1 clinical trials. The New England Journal of Medicine, 358, 2316-2317. doi:10.1056/NEJMp0801872

Emanuel, E. J. (2005). Undue inducement: Nonsense on stilts? American Journal of Bioethics, 5(5), 9-13; discussion W8-11, W17. doi:10.1080/15265160500244959

Emanuel, E. J., Currie, X. E., Herman, A., \& Project, P. (2005). Undue inducement in clinical research in developing countries: Is it a worry? The Lancet, 366, 336-340. doi:10.1016/ S0140-6736(05)66992-9

Fry, C. L., Ritter, A., Baldwin, S., Bowen, K. J., Gardiner, P., Holt, T., . . Johnston, J. (2005). Paying research participants: A study of current practices in Australia. Journal of Medical Ethics, 31, 542-547. doi:10.1136/jme.2004.009290

Gelinas, L., Largent, E. A., Cohen, G., Kornetsky, S., Bierer, B. E., \& Lynch, H. F. (2018). A framework for ethical payment to research participants. The New England Journal of Medicine, 378, 766-771.

Grady, C. (2001). Money for research participation: Does it jeopardize informed consent? American Journal of Bioethics, 1, 40-44.

Grady, C. (2005). Payment of clinical research subjects. Journal of Clinical Investigation, 115, 1681-1687. doi:10.1172/ JCI25694

Grady, C., Dickert, N., Jawetz, T., Gensler, G., \& Emanuel, E. (2005). An analysis of U.S. practices of paying research participants. Contemporary Clinical Trials, 26, 365-375. doi:10.1016/j.cct.2005.02.003

Iltis, A. S., Matsuo, H., \& DeVader, S. R. (2008). Ethical and practical concerns in developing payment policies for research involving children and adolescents. The Journal of Law, Medicine \& Ethics, 36, 413-418.

Largent, E. A., \& Lynch, H. F. (2017). Paying research participants: The outsized influence of "undue influence." IRB, 39(4), 1-9. Retrieved from https://www.ncbi.nlm.nih.gov $/$ pubmed/29038611

Latterman, J., \& Merz, J. F. (2001). How much are subjects paid to participate in research? American Journal of Bioethics, 1, 45-46. doi:10.1162/152651601300169040

Lynch, H., Joffe, S., Thirumurthy, H., Xie, D., \& Largent, E. (2019). Association between financial incentives and participant deception about study eligibility. JAMA Network Open, 2(1), e187355.

Macklin, R. (1981). On paying money to research subjects: "Due" and "undue" inducements. IRB, 3(5), 1-6. Retrieved from https://www.ncbi.nlm.nih.gov/pubmed/11649367
Marathe, P. A., Tripathi, R. K., Shetty, Y. C., Kuyare, S. S., Kamat, S. K., \& Thatte, U. M. (2018). Payment for participation in clinical research: Review of proposals submitted to the ethics committees. Perspectives in Clinical Research, 9, 64-69. doi:10.4103/picr.PICR_159_16

Mweemba, C., Ali, J., \& Hyder, A. A. (2018). Providing monetary and non-monetary goods to research participants: Perspectives and practices of researchers and Research Ethics Committees in Zambia. Global Bioethics. Retrieved from https://www. tandfonline.com/action/showCitFormats?. doi:10.1080/1128 7462.2018 .1527672

Office for Human Research Protections. (2016). OHRP expedited review categories (1998). Rockville, MD: U.S. Department of Health \& Human Services.

Phillips, T. B. (2011). A living wage for research subjects. The Journal of Law, Medicine \& Ethics, 39, 243-253. doi:10.1111/ j.1748-720X.2011.00593.x

Resnik, D. B. (2015). Bioethical issues in providing financial incentives to research participants. Medicolegal and Bioethics, 5, 35-41. doi:10.2147/MB.S70416

Ripley, E. (2006). A review of paying research participants: It's time to move beyond the ethical debate. Journal of Empirical Research on Human Research Ethics, 1(4), 9-20. doi:10.1525/ jer.2006.1.4.9

Ripley, E., Macrina, F., Markowitz, M., \& Gennings, C. (2010a). Who's doing the math? Are we really compensating research participants? Journal of Empirical Research on Human Research Ethics, 5(3), 57-65. doi:10.1525/jer.2010.5.3.57

Ripley, E., Macrina, F., Markowitz, M., \& Gennings, C. (2010b). Why do we pay? A national survey of investigators and IRB chairpersons. Journal of Empirical Research on Human Research Ethics, 5(3), 43-56. Retrieved from https://www.ncbi .nlm.nih.gov/pubmed/20831420. doi:10.1525/jer.2010.5.3.43

Ross, L. F. (2005). Payment in pediatric research. Journal of Medicine and Law, 9, 1-24.

Shamoo, A. E., \& Resnik, D. B. (2006). Strategies to minimize risks and exploitation in phase one trials on healthy subjects. American Journal of Bioethics, 6(3), W1-13. doi:10.1080 /15265160600686281

Singer, E., \& Couper, M. P. (2008). Do incentives exert undue influence on survey participation? Experimental evidence. Journal of Empirical Research on Human Research Ethics, 3(3), 49-56. doi:10.1525/jer.2008.3.3.49

Taplin, S., Chalmers, J., Hoban, B., McArthur, M., Moore, T., \& Graham, A. (2019). Children in social research: Do higher payments encourage participation in riskier studies? Journal of Empirical Research on Human Research Ethics, 14, 126-140. doi:10.1177/1556264619826796

Viens, A. M. (2001). Socio-economic status and inducement to participate. American Journal of Bioethics, 1(2), 1f-2f. doi:10.1162/152651601300169202

Wendler, D., Rackoff, J. E., Emanuel, E. J., \& Grady, C. (2002). The ethics of paying for children's participation in research. The Journal of Pediatrics, 141, 166-171. doi:10.1067/mpd.2002.124381

Wong, J. C., \& Bernstein, M. (2011). Payment of research subjects for more than minimal risk trials is unethical. The American Journal of the Medical Sciences, 342, 294-296. doi:10.1097/ MAJ.0b013e318227e0a1 


\section{Author Biographies}

Brandon Brown is an associate professor of medicine at the University of California, Riverside. His interest in the ethics of payment in research arose from his experience conducting clinical trials. He supervised the stellar graduate student who collected the data for this manuscript and provided guidance in manuscript preparation.

Logan Marg is a sociology $\mathrm{PhD}$ candidate at the University of California, Riverside. He has worked on numerous research projects related to the ethics of payment in research with Dr. Brown. He collected the data, contributed to the analysis, and drafted the manuscript.

Zhiwei Zhang is an associate professor of biostatistics at the University of California, Riverside. He is interested in developing statistical methods for biomedical applications, including causal inference and precision medicine. He contributed to the analysis and interpretation of data in the present research.

Dario Kuzmanović is a research ethics enthusiast with over 20 years of experience in research. He currently works at University of
California and is a member of the Joint Centre for Bioethics. As a research ethicist, he has consulted for numerous private companies and international groups, including the World Health Organization. In the present research, he assisted with study design, data analysis, and manuscript preparation.

Karine Dubé is an assistant professor in the Public Health Leadership Program (PHLP) in the Gillings School of Global Public Health at the University of North Carolina at Chapel Hill (UNC-CH). She works with Dr. Brandon Brown on the ethics of incentives in HIV research. She reviewed the paper for intellectual contents.

Jerome Galea is an assistant professor in the School of Social Work at the University of South Florida and collaborates on projects investigating of the impact of incentives on recruitment and retention of research participants. In particular, he is interested in how incentives impact participation in clinical research that involves greater than minimal risk to subjects, including some types of HIV treatment projects. In the research reported, he participated with the research team in the development of the study instrument, data analysis, and manuscript preparation. 